\title{
Thermodynamics and Van der Waals phase transition of charged black holes in flat spacetime via Rényi statistics
}

\author{
Chatchai Promsiri $\oplus^{1,2, *}$ Ekapong Hirunsirisawat $\odot,{ }^{2,3, \dagger}$ and Watchara Liewrian $\oplus^{1,2,4, \ddagger}$ \\ ${ }^{1}$ Department of Physics, King Mongkut's University of Technology Thonburi, \\ Pracha Uthit Road, Bangkok 10140, Thailand \\ ${ }^{2}$ Theoretical and Computational Physics (TCP), Theoretical and Computational Science Center (TaCS), \\ King Mongkut's University of Technology Thonburi, Pracha Uthit Road, Bangkok 10140, Thailand \\ ${ }^{3}$ Learning Institute, King Mongkut's University of Technology Thonburi, \\ Pracha Uthit Road, Bangkok 10140, Thailand \\ ${ }^{4}$ Thailand Center of Excellence in Physics, Ministry of Higher Education, Science, \\ Research and Innovation, 328 Si Ayutthaya Road, Bangkok 10400, Thailand
}

(Received 13 June 2020; accepted 5 August 2020; published 9 September 2020)

\begin{abstract}
The phase structure and critical phenomena of the $3+1$ dimensional charged black holes in asymptotically flat spacetime are investigated within the Rényi statistics. As the nonextensive parameter $\lambda$ above zero, a charged black hole can be in thermodynamic equilibrium with surrounding thermal radiation and have a Hawking-Page phase transition. This gives more evidence supporting the proposed conjectured equivalence between the black hole thermodynamics in asymptotically flat spacetime via Rényi statistics and that in asymptotically anti-de Sitter (AdS) spacetime via Gibbs-Boltzmann statistics. The present work also provides another aspect of supporting evidence through exploring the extended phase space within the Rényi statistics. Working on a modified version of the Smarr formula, the thermodynamic pressure $P$ and volume $v$ of a charged black hole are found to be related to $\lambda$. The thermodynamics of asymptotically flat charged black holes via Rényi statistics has the Van der Waals phase structure, $P-v$ criticality and universal constant, in a similar way as that of asymptotically AdS charged black hole via Gibbs-Boltzmann statistics. This raises an interesting question of how $\lambda$ in the former system relates to $|\Lambda|$ in the latter one.
\end{abstract}

DOI: 10.1103/PhysRevD.102.064014

\section{INTRODUCTION AND MOTIVATIONS}

In general relativity, a black hole was initially expected to be a dark object as a result of the existence of the event horizon inside, which nothing can escape. However, according to black hole thermodynamics initiated by Bekenstein [1] and Hawking [2], a black hole can have entropy and nonzero temperature. Later on there have been further works, namely the laws of black hole mechanics, suggesting that the area and the surface gravity of a black hole's event horizon correspond to entropy and temperature, respectively [3]. Furthermore, the electrostatic potential and angular velocity at the event horizon can be treated as the chemical potentials. Surprisingly, the mathematical

*chatchaipromsiri@gmail.com

†ekapong.hir@mail.kmutt.ac.th

watchara.liewrian@mail.kmutt.ac.th

Published by the American Physical Society under the terms of the Creative Commons Attribution 4.0 International license. Further distribution of this work must maintain attribution to the author(s) and the published article's title, journal citation, and DOI. Funded by SCOAP. analogy between the laws of black hole mechanics and of thermodynamics has led us to the notion that a black hole could behave as a thermal object.

Usually, a zero-charge black hole in an asymptotically flat spacetime background can only be in the phase with negative heat capacity at an arbitrary temperature, such that it cannot be in a thermal equilibrium with a heat bath of radiation. However, a rich phase structure can be found in some spacetime backgrounds through thermodynamic stability analysis. For instance, in an asymptotically anti-de Sitter (AdS) space there are possibly two branches of uncharged black holes, namely the small and large black holes. The small black hole phase has negative heat capacity implying that it is thermodynamically unstable. On the other hand, the large black hole phase has positive heat capacity, hence it is thermodynamically stable. However, these two black hole phases can exist only above a certain temperature, let us call it $T_{\min }$ here. Below $T_{\min }$, the pure thermal radiation bath occupies the AdS space. Intriguingly, there is the HawkingPage phase transition at the temperature $T_{\mathrm{HP}}$, slightly above $T_{\min }$. At the temperature in the range between $T_{\min }$ and $T_{\mathrm{HP}}$, the thermal radiation is mostly thermodynamically preferred over the small and large black hole phases. The large black 
hole phase turns out to be the most thermodynamically preferred at $T>T_{\mathrm{HP}}$ [4].

There have been some controversial issues for a long time, such as whether it is appropriate to apply the standard Gibbs-Boltzmann (GB) approach to a self-gravitating system. Gravitation is a long-range attractive force, such that the average potential energy $\langle V\rangle$ between particles in a self-gravitating system is negative. Given that $\langle K\rangle$ is a positive value of average kinetic energy, we can use the virial theorem to show that the total average energy of the system $\langle U\rangle$ has negative value, namely, $\langle U\rangle=\langle K\rangle+$ $\langle V\rangle=-\langle K\rangle$. It is well known that the kinetic energy $\langle K\rangle$ is linear in the temperature $T$ up to some constants due to the equipartition theorem. As a consequence, we obtain the heat capacity as $C=d\langle U\rangle / d T<0[5,6]$. Importantly, a negative heat capacity indicates that the self-gravitating system is thermodynamically unstable. This implies that the system cannot become thermodynamically equilibrium when it is in thermal contact with a heat bath. While this is a weird behavior of the self-gravitating system, it might be possible that it is an incorrect conclusion. A complete understanding of this situation has still been a challenging issue. Generically, the standard GB statistical approach should be violated in the case of long-range interactions due to some clues such as the existence of a divergent partition function. This has been pointed out by Gibbs [7] and later on by others [8-10] (see also [11] and references therein). In other words, applying the standard GB approach in the case of a self-gravitating system may lead us to obtain an incomplete result.

In conventional thermodynamic systems of ordinary matter, the entropy of a whole system can be written as the sum of the entropy of subsystems. In this way, the entropy of the system typically scales with its volume. As a result, it is said to be an "extensive variable." However, Bekenstein argued that a black hole system carries entropy proportional to the surface area of its event horizon rather than the volume. Such behavior is called an "area law." Therefore, the black hole's entropy is nonextensive. Intriguingly, this black hole area law guides us to the holographic principle, which states that the information in a higher dimensional bulk spacetime can be encoded into its boundary $[12,13]$. Later on, this principle becomes more established by the developments of the AdS/CFT correspondence [14-16]. Moreover, the area law can be found in the entanglement entropy of the reduced state of a subregion in strongly correlated quantum systems [17]. This similarity raises the question about whether the quantum origin of black hole entropy may be somehow related to the entanglement entropy. Currently, the nonextensive nature of entropy has received wide attention in several fields, and it might possibly improve our insights about the microscopic nature of black holes.

As discussed above, the GB statistics might not be appropriate to use in black hole thermodynamics. In other words, the black hole entropy $S_{\mathrm{BH}}$ should encode the black hole information with nonlocal and nonextensive nature. Consequently, we need a non-Boltzmannian approach to deal with this. A new type of entropic function can be introduced by relaxing the Shannon-Khinchin axiomatic definition of the entropic function, i.e., additivity, to the weaker nonadditive composition rule. With composability, the entropic function can remain to be physically meaningful. According to the derivation of Abe, the most general nonadditive entropy composition rule is in the form [18]

$$
H_{\lambda}\left(S_{12}\right)=H_{\lambda}\left(S_{1}\right)+H_{\lambda}\left(S_{2}\right)+\lambda H_{\lambda}\left(S_{1}\right) H_{\lambda}\left(S_{2}\right),
$$

where $H_{\lambda}$ is a differentiable function of $S$, and $\lambda \in \mathbb{R}$ is a constant parameter. One of the simplest versions of nonextensive entropy, obeying Tsallis entropy, can be written in the form [10]

$$
S_{T}=\frac{1}{1-q}\left(\sum_{i=1}^{W} p_{i}^{q}-1\right)
$$

where $p_{i}$ are the probabilities of microstates of the system, $\mathrm{W}$ is the total number of microstates, and $q \in \mathbb{R}$ is the dimensionless parameter of nonextensivity. Clearly, the standard GB entropy is recovered when $q \rightarrow 1$. The composition rule of nonadditive Tsallis entropy can be written as

$$
S_{T}^{12}=S_{T}^{1}+S_{T}^{2}+(1-q) S_{T}^{1} S_{T}^{2}
$$

which satisfies the Abe's nonadditive entropy composition rule, as shown in (1), when $H_{\lambda}(S)=S_{T}$ and $\lambda=1-q$. However, there is a long-standing problem about the thermal equilibrium for nonextensive systems relating to the compatibility with the zeroth law of thermodynamics. Namely, if two systems are in the thermal equilibrium, then the total entropy has the maximum value $d S_{A B}=d\left(S_{A}+S_{B}\right)=0$, which implies the existence of an empirical temperature $\frac{1}{T}=\frac{\partial S_{A}}{\partial E_{A}}=\frac{\partial S_{B}}{\partial E_{B}}$. The problem with nonextensive entropy is that its composition rule is in the nonadditive form, hence it is not clear whether we can define an empirical temperature [19]. Recently, Biró and Ván proposed a way to solve this problem by transforming the nonadditive entropy into another one that has an additive composition rule, which satisfies the zeroth law of thermodynamics [20]. Their method is called the "formal logarithmic approach." They showed that, for a homogeneous system, the Tsallis entropy can be transformed into the well-defined entropy function as

$$
L\left(S_{T}\right)=\frac{1}{1-q}\left[\ln \left(1+(1-q) S_{T}\right)\right] \equiv S_{R}
$$

Interestingly, this result is a well-known Rényi entropy $S_{R}$ defined up to an arbitrary real parameter $q$ as [21] 


$$
S_{R}=\frac{1}{1-q} \ln \sum_{i=1}^{W} p_{i}^{q}
$$

where $0<q<\infty$ and reduces to the standard GB entropy as $q$ approaches to 1 . Notice that the composition rules of the Rényi entropy for independent subsystems are in the additive form:

$$
S_{R}^{12}=S_{R}^{1}+S_{R}^{2}
$$

Now, the entropy function is compatible with the zeroth law of thermodynamics, and then we can uniquely define an empirical temperature in thermal equilibrium among subsystems as

$$
\frac{1}{T_{R}}=\frac{\partial S_{R}(E)}{\partial E},
$$

where $E$ is the energy of the system.

The non-Boltzmannian approach has been applied to investigate the black hole entropy problem in [22] and references therein. Recently, the thermodynamic stability of Schwarzschild and Kerr black holes in asymptotically flat spacetime were investigated via Rényi statistics in [23,24]. Interestingly, their works have shown that the black holes in asymptotically flat spacetime can be in a stable equilibrium with the heat bath at a fixed temperature ensemble at a positive nonextensive parameter $\lambda=1-q$. Moreover, the black holes in Rényi's model can have a small/large black hole first-order phase transition in the same way as the Hawking-Page phase transition of black holes in AdS space with the conventional GB statistics. Although the connection between these two pictures has been shown and claimed in previous works, this cannot be shown in a convincing way. It is interesting to raise a question about how the $\lambda$ parameter relates to the cosmological constant $\Lambda$ of AdS space. We will explore this in the case of a charged black hole.

In conventional thermodynamics, the phase structure of the asymptotically AdS Reissner-Nordström black holes (RN-AdS) has been investigated in both grand canonical (fixed potential) and canonical (fixed charged) ensembles, see [25-27]. In particular, RN-AdS black holes in a canonical ensemble have been found to thermodynamically behave in a similar way as the Van der Waals (VdW) liquidgas system. Recently, more concrete comparison can be achieved by identifying thermodynamic pressure $P$, specific volume $v$, and temperature $T$ in the $\mathrm{VdW}$ system with the cosmological constant $\Lambda$, the black-hole horizon radius $r_{+}$, and the Hawking temperature $T_{H}$ of the RN-AdS black hole, respectively. We can treat the RN-AdS black hole as a chemical system with

$$
P=-\frac{\Lambda}{8 \pi}=\frac{3}{8 \pi L^{2}}, \quad v=2 l_{p}^{2} r_{+}, \quad T=T_{H},
$$

where the cosmological constant $\Lambda=-\frac{3}{L^{2}}$, and $L$ is the AdS radius. The consideration in this way is called the "extended phase space" [28-30]. With this approach, there are a number of investigations of black hole thermodynamics and phase transitions in many spacetime backgrounds and modified gravitational theories [31-33]. Moreover, the fascinating studies on critical phenomena and microstructure of black holes using the thermodynamic geometry can be seen in [34-36]. It is interesting to note that the VdW-like behavior of five-dimensional charged black holes in AdS from hysteresis in the context of the AdS/CFT correspondence has been studied in [37]. This could give us some hints to find a way for applying this in our real-world phenomena.

In the present paper, being motivated by the nonextensive nature of black hole entropy and the VdW-like phase structure of a charged black hole in the AdS background in the extended phase space approach, we explore the thermodynamics of the $3+1$ dimensional ReissnerNordström black hole in asymptotically flat spacetime (RN-flat) through Rényi statistics, and then investigate its phase structure in the extended phase space. To achieve this, we need to modify the Smarr relation in terms of Rényi entropy and its corresponding empirical temperature. With this approach, the thermodynamic pressure $P$ is found to relate to the nonextensive parameter $\lambda$. Interestingly, as will be shown, this relation between $P$ and $\lambda$ in the modified extended phase space of the RN-flat via the Rényi statistics corresponds to the relation between $P$ and the cosmological constant $|\Lambda|$ in the extended phase space approach in the RN-AdS setup via the GB statistics, as shown in (8). Furthermore, the VdW-like phase transition has also been found in this setting. These give us more strong evidence about the connection between thermodynamics of black holes in AdS space calculated from GB statistics and black holes in flat space calculated from Rényi statistics, as proposed in $[23,24]$. We discuss this connection in this paper.

The organization of the paper is as follows. In Sec. II, we review the spherical charged black hole solution in asymptotically flat spacetime and discuss its standard thermodynamics in fixed potential and fixed charge ensemble. In Sec. III, the thermodynamic properties of the $\mathrm{RN}$-flat are investigated within the Rényi statistics. The thermal phase structure is also studied in this section. Then, we suggest in Sec. IV, an analogous thermal behavior of the charged black hole in Rényi statistics to the Van der Waals liquidgas system. The concluding remarks and the suggestion for further studies are discussed in Sec. V.

\section{REVIEW OF CHARGED BLACK HOLE THERMODYNAMICS}

In this section, we review the standard thermodynamics of the $3+1$ dimensional RN-flat spacetime. Starting with a spherically symmetric Reissner-Nordström metric of mass $M$ and charged $Q$ of the form 


$$
d s^{2}=-f(r) d t^{2}+\frac{d r^{2}}{f(r)}+r^{2} d \Omega_{2}^{2}
$$

where $d \Omega_{2}^{2}=d \theta^{2}+\sin ^{2} \theta d \phi^{2}$ is the square of line element on 2 -sphere and the function $f(r)$ is given by

$$
f(r)=1-\frac{2 M}{r}+\frac{Q^{2}}{r^{2}} .
$$

The black hole horizon can be determined from the condition $f(r)=0$, where its roots consist of

$$
r_{ \pm}=M \pm \sqrt{M^{2}-Q^{2}},
$$

where $r_{+}$and $r_{-}$are the radii of the outer and inner horizon, respectively. The black hole horizon is at $r_{+}$, from which the Hawking radiation is generated. The black hole mass $M$ relates to the event horizon radius and the charge $Q$ as follows:

$$
M=\frac{r_{+}}{2}\left(1+\frac{Q^{2}}{r_{+}^{2}}\right)
$$

The charge $Q$ can generate the gauge field of the form

$$
A=A_{t} d t=-\left(\frac{Q}{r}-\Phi\right) d t
$$

By setting $A_{t}=0$ at the horizon, we can relate the electric potential $\Phi$ with $M$ and $Q$ using $r_{+}=M+\sqrt{M^{2}-Q^{2}}$. Thus, we have

$$
\Phi=\frac{Q}{r_{+}}=\frac{Q}{M+\sqrt{M^{2}-Q^{2}}} .
$$

Obviously, the outer and inner horizons are degenerate when $M=Q$. Namely, this extremal black hole has only one horizon, i.e., $r_{e}=r_{+}=r_{-}$. On the other hand, the charged black hole has no horizon when $M<Q$. To avoid this condition of naked singularity, the mass of the black hole must not be smaller than its charge, i.e., $M \geq Q$, therefore, the electric potential is allowed to have the value in the range $0 \leq \Phi \leq 1$.

In black hole thermodynamics, physical quantities in a black hole system can be treated as thermodynamic variables. This results from the mathematical analogy between the laws of black hole mechanics and the laws of thermodynamics. The first law of black hole mechanics relates two stationary black holes with the change in mass $\delta M$, the horizon surface area $\delta A$, the angular momentum $\delta J$, and electric charge $\delta Q$ via the relation [3]

$$
\delta M=\frac{\kappa}{8 \pi G} \delta A+\Omega \delta J+\Phi \delta Q,
$$

where $\kappa, \Omega$, and $\Phi$ are surface gravity, angular velocity, and electric potential at the event horizon, respectively. This can be seen as the first law of thermodynamics when one identifies the event horizon area $A$, surface gravity $\kappa$ with entropy, and temperature of the black hole, respectively. For a nonrotating charged black hole, $Q$ is the number of particles in thermodynamic description since the charge simply counts the number of particles, and its conjugate $\Phi$ plays a role of chemical potential.

\section{A. Grand canonical ensemble}

One can consider thermodynamics of black holes in either grand canonical or canonical ensemble. When the black hole exchanges charge $Q$ with the surrounding heat bath, the chemical potential $\Phi$ can be held to be fixed. In this way, the system is being considered in the grand canonical ensemble. Consequently, the charged black hole thermodynamic quantities can be written as

$$
\begin{gathered}
E=M=\frac{r_{+}\left(1+\Phi^{2}\right)}{2}, \\
T_{H}=\frac{f^{\prime}\left(r_{+}\right)}{4 \pi}=\frac{1-\Phi^{2}}{4 \pi r_{+}}, \\
S_{\mathrm{BH}}=\frac{A}{4}=\pi r_{+}^{2}, \\
C_{\Phi}=T_{H}\left(\frac{\partial S_{\mathrm{BH}}}{\partial T_{H}}\right)_{\Phi}=-\frac{2 \pi r_{+}^{2}\left(1+\Phi^{2}\right)}{1-\Phi^{2}}, \\
G=E-T_{H} S_{\mathrm{BH}}-\Phi Q=\frac{r_{+}\left(1-\Phi^{2}\right)}{4},
\end{gathered}
$$

where $E$ is the internal energy, $T_{H}$ is the Hawking temperature, $S_{\mathrm{BH}}$ is the Bekenstein-Hawking entropy, $C_{\Phi}$ is the heat capacity at a fixed electric potential $\Phi$, and $G$ is the Gibbs free energy, respectively. Because the heat capacity $C_{\Phi}$ is negative at an arbitrary value of $r_{+}$and $\Phi$, the black hole phase cannot be in a stable equilibrium with pure thermal radiation in the grand canonical ensemble. This is shown as a black dashed line in Figs. 1 and 2.

\section{B. Canonical ensemble}

Working in the canonical ensemble, we consider a black hole at fixed charge $Q$. In this case, we measure the energy $E$ of the system with respect to the ground state, which is the extremal black hole. The mass $M_{e}$ of the extremal black hole can be obtained from the condition that the $r_{+}$(outer) and $r_{-}$(inner) horizon radii are degenerated. Hence, the mass and charge of an extremal black hole are expressed as $M_{e}=Q=r_{e}$. Therefore, the thermodynamic quantities in the canonical ensemble take the form 

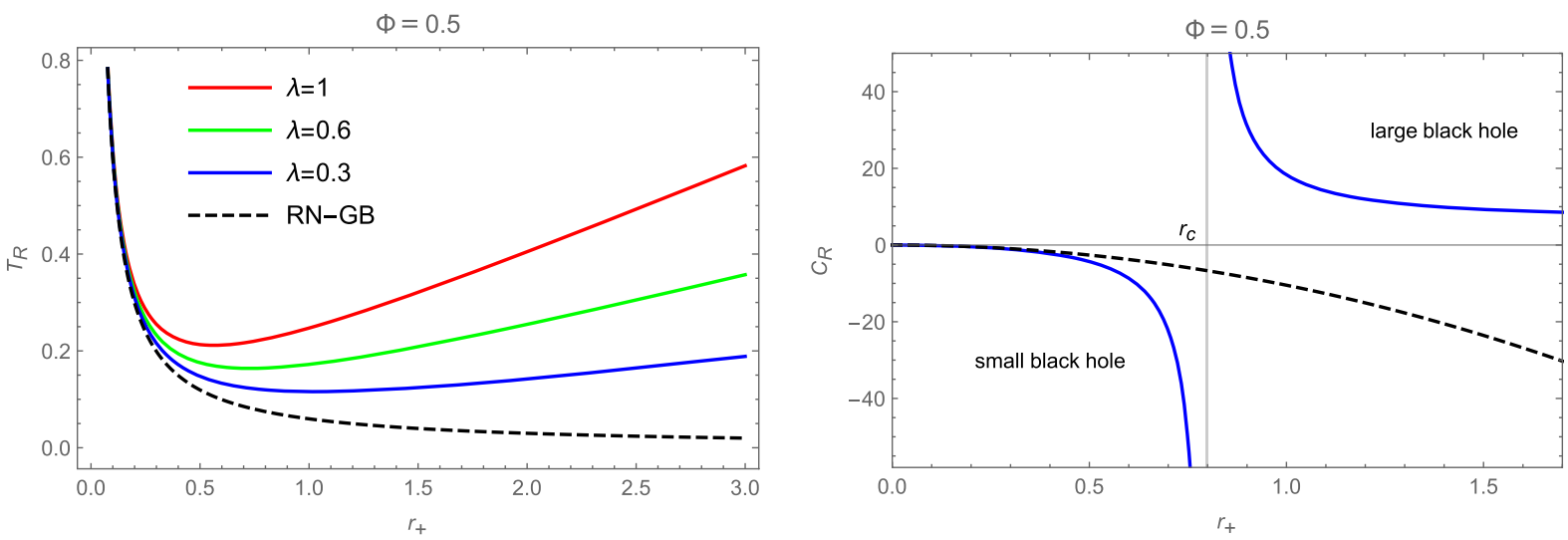

FIG. 1. Left: Rényi temperature of a charged black hole $T_{R}$ versus the event horizon radius $r_{+}$for a fixed value $\Phi=0.5$ is plotted with $\lambda=0.3$ (solid blue), 0.6 (solid green), and 1.0 (solid red), compared with the case of the GB statistics, $\lambda=0$ (dashed black). Right: heat capacity $C_{R}$ of a charged black hole versus $r_{+}$with $\Phi=0.5$ is plotted in the case of $\lambda=0.3$ (solid blue), its value is negative at $r_{+}<r_{c}$, while it is positive at $r_{+}>r_{c}$. However, the heat capacity cannot be positive at all values of $r_{+}$in the GB statistics, $\lambda=0$ (dashed black).

$$
\begin{gathered}
E=M-M_{e}=\frac{\left(r_{+}-Q\right)^{2}}{2 r_{+}}, \\
T_{H}=\frac{f^{\prime}\left(r_{+}\right)}{4 \pi}=\frac{r_{+}^{2}-Q^{2}}{4 \pi r_{+}^{3}}, \\
S_{\mathrm{BH}}=\frac{A}{4}=\pi r_{+}^{2}, \\
C_{Q}=T_{H}\left(\frac{\partial S_{\mathrm{BH}}}{\partial T_{H}}\right)_{Q}=-\frac{2 \pi r_{+}^{2}\left(r_{+}^{2}-Q^{2}\right)}{r_{+}^{2}-3 Q^{2}}, \\
F=E-T_{H} S_{\mathrm{BH}}=\frac{\left(r_{+}-Q\right)\left(r_{+}-3 Q\right)}{4 r_{+}},
\end{gathered}
$$

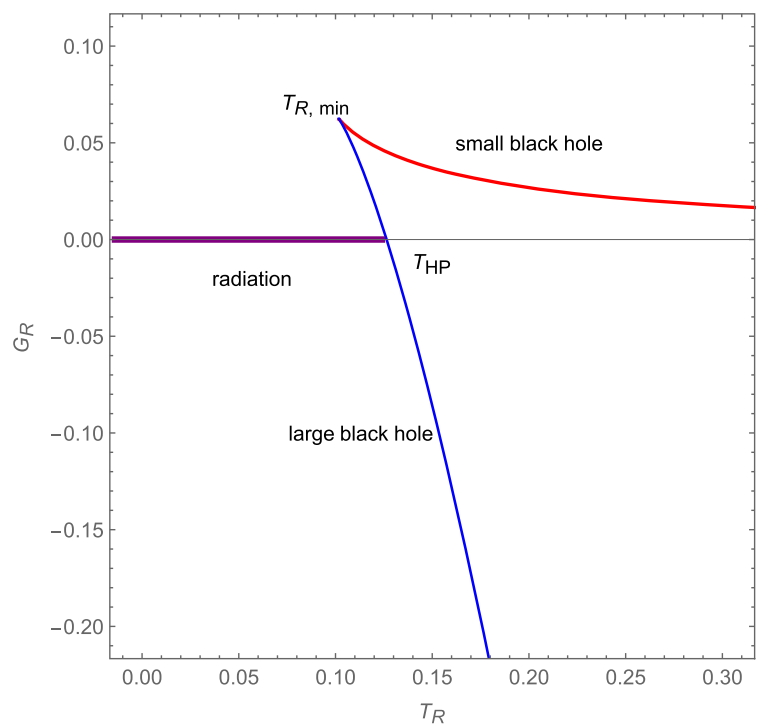

where all thermodynamic variables are defined as in the grand canonical ensemble, except that $C_{Q}$ here is the heat capacity at a constant number of particles, and $F$ is the Helmholtz free energy. From (24), we have two branches of charged black holes in which each black hole has either positive or negative heat capacity. The heat capacity is positive when

$$
Q<r_{+}<\sqrt{3} Q
$$

whereas, it is negative when $r_{+}>\sqrt{3} Q$. At the critical point $r_{c}=\sqrt{3} Q$, the heat capacity diverges as the Hawking temperature reaches its maximum value

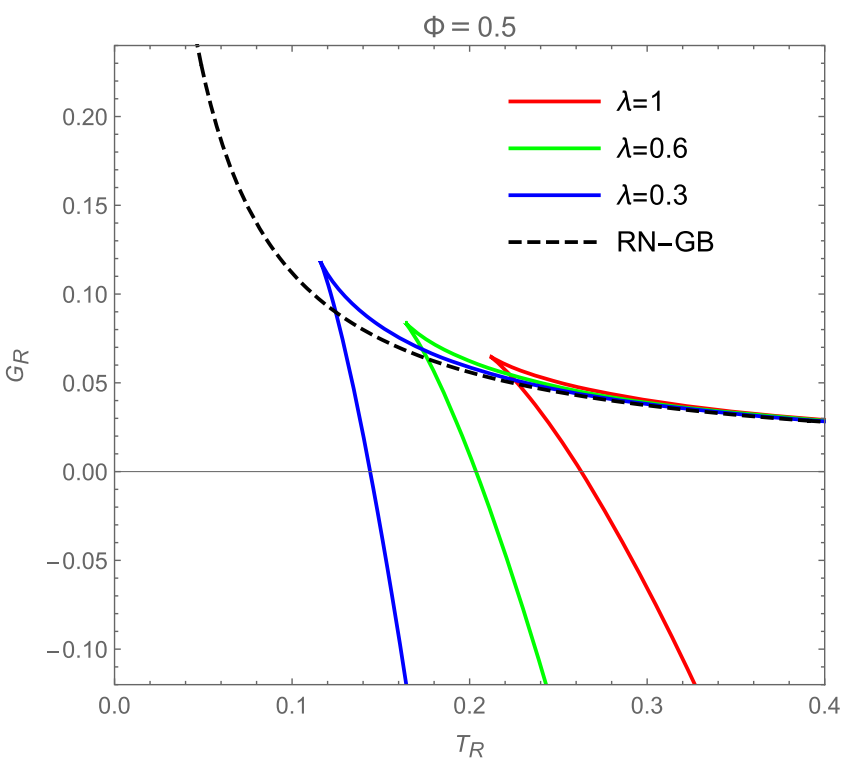

FIG. 2. Left: Gibbs free energy vs temperature for fixed $\Phi=0.5$ in the Rényi entropy. Right: Gibbs free energy vs temperature by varying Rényi parameters as $\lambda=0.3,0.6$ and 1 corresponding to blue, green, and red curves, respectively. The plot from the GB statistics is shown in this graph with black dashed curve. 


$$
T_{\max }=\frac{1}{6 \sqrt{3} \pi Q}
$$

In contrast with typical systems, the phase transition of RNflat black holes depends on the ensemble in consideration. This might result from the long-range nature of gravitational and electromagnetic interactions of the system [38].

\section{THERMODYNAMICS AND THERMAL PHASE TRANSITION VIA RÉNYI STATISTICS}

Now, we turn to an alternative approach to study thermodynamic properties of charged black holes. Here, we use the Rényi statistics and then calculate thermodynamic quantities. Then, we will investigate the thermal phase structure of RN-flat black holes in both the fixed potential and fixed charge ensemble.

As suggested in previous works [23,24], black holes can be treated in the way that follows the nonadditive Tsallis statistics, whose composition rule is in the simplest form as shown in (3). Even though the Tsallis entropy function tends to be one of the proper choices representing black hole entropy, it turns out to have difficulty defining the empirical temperature through the zeroth law of thermodynamics. To avoid this, the Rényi entropy in the form of the formal logarithm of the Tsallis entropy is proposed [20]. Using this transformation rule, we obtain the Rényi entropy function of a black hole as

$$
S_{R}=\frac{1}{\lambda} \ln \left(1+\lambda S_{\mathrm{BH}}\right),
$$

which has an additive property. The Rényi temperature can be expressed as

$$
T_{R}=\frac{1}{\partial S_{R} / \partial M}=T_{H}\left(1+\lambda S_{\mathrm{BH}}\right)
$$

Based on this formula of the Rényi entropy and its corresponding temperature, we consider in this section the thermal phase transitions of $\mathrm{RN}$-flat black holes in the grand canonical and the canonical ensemble.

\section{A. Grand canonical ensemble}

For the grand canonical ensemble with a fixed value of $\Phi$, the corresponding Rényi temperature as a function of the event horizon $r_{+}$and the electrical potential $\Phi$ is given by

$$
T_{R}=\frac{\left(1-\Phi^{2}\right)\left(1+\lambda \pi r_{+}^{2}\right)}{4 \pi r_{+}} .
$$

The heat capacity can be obtained in a usual way

$$
C_{R}=T_{R}\left(\frac{\partial S_{R}}{\partial T_{R}}\right)_{\Phi}=-\frac{2 \pi r_{+}^{2}\left(1+\Phi^{2}\right)}{\left(1-\Phi^{2}\right)\left(1-\lambda \pi r_{+}^{2}\right)} .
$$

Obviously, the heat capacity is negative when $r_{+}<r_{c}$ and positive when $r_{+}>r_{c}$, where $r_{c}=\sqrt{1 / \lambda \pi}$. Accordingly, there are two possible black hole configurations: one with negative value of heat capacity and another with positive value. We will refer to these as small and large black holes, respectively. Substituting $r_{c}$ into (12), we obtain the critical mass

$$
M_{c}=\frac{1+\Phi^{2}}{2 \sqrt{\lambda \pi}} .
$$

In Rényi statistics, a RN-flat black hole is in the small black hole branch when $M<M_{c}$ and in the large black hole branch when $M>M_{c}$.

The heat capacity $C_{R}$ is inversely proportional to the slope of the graph $T_{R}$ versus $r_{+}$, as shown in Fig. 1 (left). Namely, we can write

$$
C_{R}=\frac{1}{2} \frac{\left(1+\Phi^{2}\right)}{\left(1-\Phi^{2}\right)^{2}}\left(\frac{1}{\partial T_{R} / \partial r_{+}}\right)
$$

Therefore, the critical radius $r_{c}$ is just a turning point at which the slope of $T_{R}\left(r_{+}\right)$changes the sign, i.e., $T_{R}^{\prime}\left(r_{+}\right)=0$. With the presence of this turning point, the RN-flat black holes with $\lambda=0.3$ (solid blue), 0.6 (solid green), and 1.0 (solid red) can be stable with positive heat capacity when $r_{+}>r_{c}$ and negative heat capacity at $r_{+}<$ $r_{c}$ while there is no turning point in the case of GB statistics (dashed black). The discussion of these results is actually equivalent to the plots in Fig. 1 (right).

Interestingly, the turning point in Fig. 1 (left) also shows that the lower bound of a Hawking temperature becomes larger with a higher level of nonextensivity. This can be seen through solving (30) for $r_{+}$, such that we have

$$
r_{L, S}=\frac{2 T_{R}}{\lambda\left(1-\Phi^{2}\right)}\left(1 \pm \sqrt{1-\frac{\lambda\left(1-\Phi^{2}\right)^{2}}{4 \pi T_{R}^{2}}}\right),
$$

where $r_{L}$ and $r_{S}$ denote the event horizon of the large and small black hole configurations at fixed temperature, respectively. The solution is real when the discriminant in the above equation is greater than zero. This implies that there is a minimum temperature at $r=r_{c}$, which is

$$
T_{R, \min }=\frac{\left(1-\Phi^{2}\right)}{2} \sqrt{\frac{\lambda}{\pi}} .
$$

Note that for the uncharged case, the minimum temperature reduces to the form $T_{R, \min }=\frac{1}{2} \sqrt{\frac{\lambda}{\pi}}$ as found in [23].

As shown in the work of Gross et al. [39], the $3+1$ dimensional hot flat space can be in the unstable state due to the nucleation of black holes without the lower bound of temperature. In other words, in the GB statistics, a black 
hole can be formed at an arbitrarily low temperature of thermal radiation. Considering this through Rényi statistics, however, gives a different conclusion. A black hole is not allowed to be created when $T_{R}<T_{R \text {,min }}$ since, as a result of that, an event horizon cannot exist due to the presence of an imaginary number in (34), and due to the fact that a naked singularity in a 4-dimensional asymptotically flat spacetime is forbidden by the cosmic censorship hypothesis [40]. Accordingly, we can argue that the hot thermal radiation in asymptotically flat spacetime can be collapsed to a black hole only when $T_{R}>T_{R \text {,min }}$. This shows that a HawkingPage phase transition of black holes in the Minkowski background is, in a similar way, what occurs in the black holes in the AdS background via GB statistics. Therefore, our result is in contrast with that derived from the consideration of Gross et al. [39].

The thermodynamic stability can be investigated through considering the free energy as a function of the temperature. In the grand canonical ensemble, we use the Gibbs free energy as thermodynamical potential. With the Rényi statistics, the Gibbs free energy function should be modified by Rényi entropy and its corresponding temperature as

$$
\begin{aligned}
G_{R} & =M-T_{R} S_{R}-\Phi Q, \\
& =\frac{r_{+}\left(1-\Phi^{2}\right)}{4}\left[2-\left(1+\frac{1}{\lambda \pi r_{+}^{2}}\right) \ln \left(1+\lambda \pi r_{+}^{2}\right)\right] .
\end{aligned}
$$

The plots of $G_{R}$ versus $T_{R}$ are shown in Fig. 2 (left). While the GB statistics $(\lambda=0)$ tell us that the charged black hole is unstable as its free energy is positive at any nonzero temperature [dashed, Fig. 2 (right)], the Rényi parameter $(\lambda>0)$ allows that the black hole can be stable above a certain temperature $T_{\mathrm{HP}}$, where the $G_{R}$ has negative value as indicated in Fig. 2 (left). From (36), we can find this temperature at which the first order Hawking-Page phase transition occurs by setting $G_{R}=0$. Numerically, we can find that

$$
T_{\mathrm{HP}} \approx 0.64\left(1-\Phi^{2}\right) \sqrt{\frac{\lambda}{\pi}}
$$

which is about 1.28 times $T_{R, \min }$. Figure 3 shows the Hawking-Page temperature as a function of the electrostatics potential. This phase transition behavior from our results are very similar to the RN-AdS via standard GB statistics [25-27]. Obviously, we can see that for $\Phi=1$ and $\lambda=0$ cases the Hawking-Page temperature vanishes, therefore, the first-order phase transition cannot exist either in the extremal black hole or in the consideration of black holes via the GB statistics.

Let us focus on the presence of the cusp of $G_{R}$ at $T_{R, \min }$, which is less than $T_{\mathrm{HP}}$. The cusp corresponds to the point of small/large black hole phase transition. This is of the second-order type due to the discontinuity of the second derivative of the Gibbs free energy with respect to the

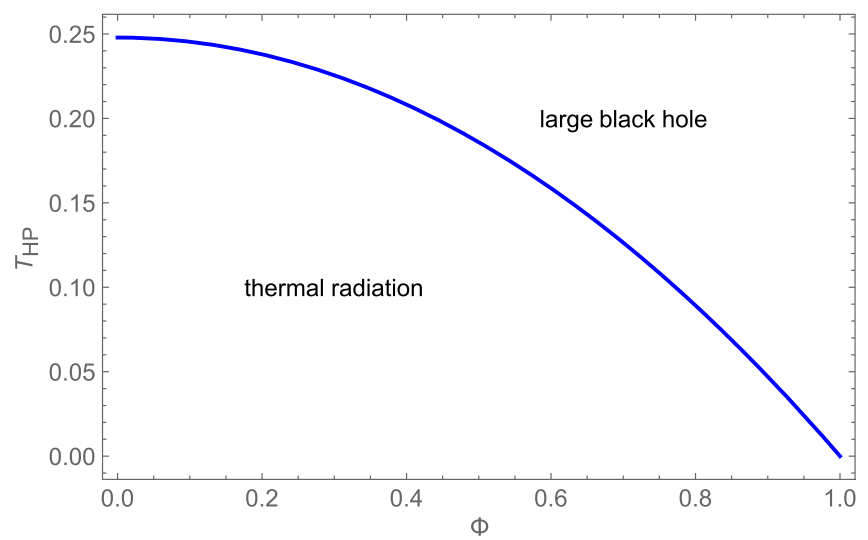

FIG. 3. Hawking-Page phase transition line in the $T-\Phi$ phase diagram. The line dividing the space into two regions, the thermal radiation phase, and large black hole phase.

temperature at the cusp, as seen clearly through the divergence of heat capacity $C_{R}=-T_{R}\left(\frac{\partial^{2} G_{R}}{\partial T_{R}^{2}}\right)$ at the cusp, as shown in Fig. 2.

$$
S_{R c}=\frac{1}{\lambda} \ln 2, \quad G_{R c}=\frac{\left(1-\Phi^{2}\right)}{2 \sqrt{\lambda \pi}}(1-\ln 2) .
$$

Notice that $S_{R c}$ is a universal constant at a fixed $\lambda$. It is obviously independent of the black hole's mass $M$ and charge $Q$. At the cusp, the Gibbs free energy is at the highest value $G_{R c}$, as shown in the equation above.

In the AdS/CFT corespondence, the electric potential $\Phi$ in the bulk has the holographic dual to the chemical potential $\mu$ of the gauge theory at the boundary. The Hawking-Page phase transition in the bulk of AdS space is dual to the phase transition between a cold confined phase and a hot deconfined phase at finite chemical potential. Intriguingly, the phase diagram from our results, as shown in Fig. 3, looks similar to the $T-\mu$ phase diagram of confining/deconfining phase transition at finite chemical potential. This provides one more supporting evidence that the physics of asymptotically flat black holes using the Rényi approach somehow corresponds to that of asymptotically AdS black holes using GB statistics, as suggested in previous works $[23,24]$.

\section{B. Canonical ensemble}

Here, we investigate the thermal properties and the phase structure of black holes in canonical ensemble (fixed $Q$ ) via Rényi statistics. Using (22) and (29) the Hawking temperature with fixed charge can be written as

$$
T_{R}=\frac{\left(r_{+}^{2}-Q^{2}\right)\left(1+\lambda \pi r_{+}^{2}\right)}{4 \pi r_{+}^{3}} .
$$

The relation between $T_{R}$ and $r_{+}$with fixed $Q=1$ at different $\lambda$ are plotted as shown in Fig. 4 (left). 

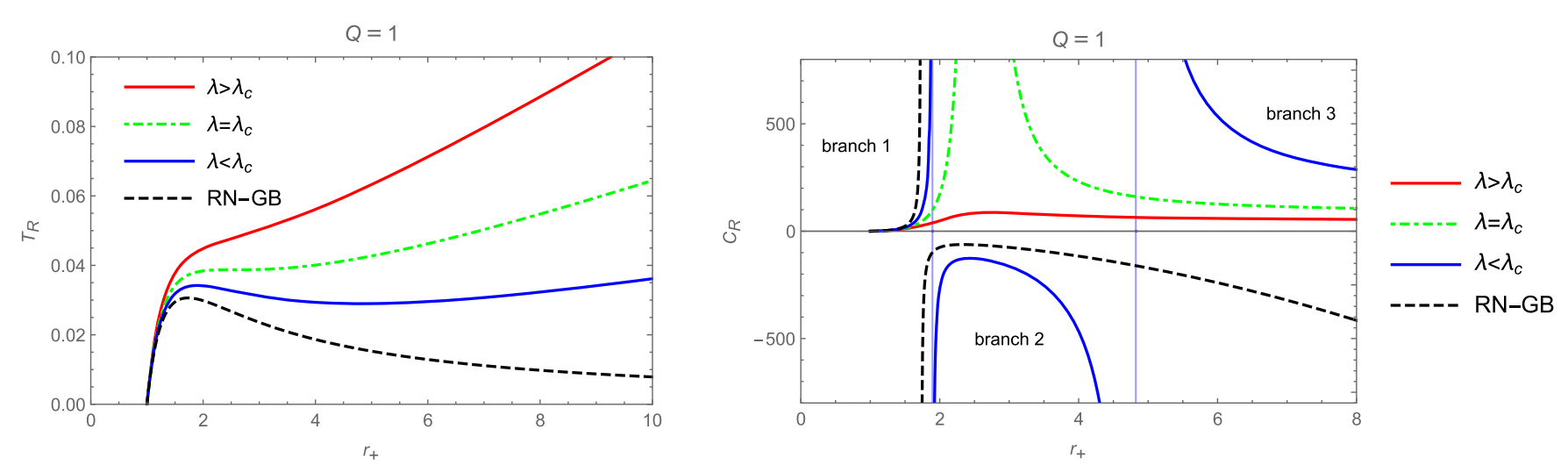

FIG. 4. Left: Plots of the Rényi temperature of charge black hole $T_{R}$ versus the event horizon radius $r_{+}$with fixed charge $Q=1$ at different value of parameter $\lambda$. Right: Heat capacity $C_{R}$ versus $r_{+}$with charge $Q=1$ at different value of $\lambda$.

These isocharge curves of the black hole temperature versus the horizon radius look similar to the case of nonzero charge black holes in AdS background [25-27]. For different values of $\lambda$ parameter, the isocharge curves behave differently where a critical phase transition can occur with the condition

$$
0=\left(\frac{\partial T_{R}}{\partial r_{+}}\right)_{Q}=\left(\frac{\partial^{2} T_{R}}{\partial r_{+}^{2}}\right)_{Q} .
$$

By solving this equation, we obtain the critical Renyi parameter $\lambda_{c}$, critical horizon $r_{c}$, and critical temperature $T_{c}$ at the critical point as

$$
\begin{gathered}
\lambda_{c}=\frac{7-4 \sqrt{3}}{\pi Q^{2}}, \\
r_{c}=(3+2 \sqrt{3})^{1 / 2} Q, \\
T_{c}=\frac{2}{(3+2 \sqrt{3})^{3 / 2} \pi Q} .
\end{gathered}
$$

For small Rényi parameter $\lambda<\lambda_{c}$, there are two local extrema of the isocharge curve in Fig. 4 (left) at

$$
r_{1,2}^{2}=\frac{1}{2 \pi \lambda}\left[\left(1-\lambda \pi Q^{2}\right) \mp \sqrt{\lambda^{2} \pi^{2} Q^{4}-14 \lambda \pi Q^{2}+1}\right],
$$

where the horizon radii $r_{1}$ and $r_{2}$ correspond to a local maximum and local minimum of the isocharge curve, respectively. The discriminant in (44) is zero as $\lambda=\lambda_{c}$, hence two extremal radii $r_{1}$ and $r_{2}$ are degenerate into $r_{c}$.

The corresponding heat capacity is

$$
C_{R}=T_{R}\left(\frac{\partial S_{R}}{\partial T_{R}}\right)_{Q}=-\frac{2 \pi r_{+}^{2}\left(r_{+}^{2}-Q^{2}\right)}{r_{+}^{2}-3 Q^{2}+\lambda \pi r_{+}^{2}\left(r_{+}^{2}+Q^{2}\right)} .
$$

In contrast with GB statistics, the Rényi approach allows three branches of charged black hole configurations in the canonical ensemble, one negative and two positive heat capacities as shown in Fig. 4 (right). We denote them as branch 1 , branch 2 , and branch 3 , respectively. The additional branch 3 now appears and shows an interesting thermal phase of the black hole. From (45), the heat capacity grows without an upper bound when the horizon radius $r_{+}$equals $r_{1}$ and $r_{2}$. The corresponding temperatures at radii $r_{1}$ and $r_{2}$ are $T_{1}=0.0299$ and $T_{2}=0.0296$, respectively, for the chosen parameters in the Fig. 4.

In the canonical ensemble, the thermodynamic potential is the Helmholtz free energy, which can be generalized to satisfy the Rényi statistics as

$$
\begin{aligned}
F_{R}= & E-T_{R} S_{R}, \\
= & \frac{Q-r_{+}}{4 \pi r_{+}^{3}}\left[2 \pi r_{+}^{2}\left(Q-r_{+}\right)\right. \\
& \left.+\frac{1}{\lambda}\left(Q+r_{+}\right)\left(1+\lambda \pi r_{+}^{2}\right) \ln \left(1+\lambda \pi r_{+}^{2}\right)\right],
\end{aligned}
$$

where $E=M-M_{e}$ is the energy of the system relative to that of the extremal black hole. Figure 5 (left) shows the relation of the free energy versus the Rényi temperature of $\mathrm{RN}$-flat black holes at a small $\lambda$ parameter. Interestingly, the swallowtail behavior occurs when $\lambda<\lambda_{c}$, which is very similar to a Van der Waals type of liquid/gas phase transition. In Fig. 5 (left), there is only branch 1 that exists at low temperature. At a certain temperature $T_{2}$, branch 2 and 3 emerge simultaneously with larger free energy than branch 1 . However, when the temperature increases to $T_{1}$, it can be seen that branch 1 and 2 combine and disappear. Just a little bit below $T_{1}$, the free energy of branch 1 and 3 are equal at temperature $T_{\mathrm{HP}}$, and the latter has more negative free energy than the first at $T>T_{\mathrm{HP}}$. This result implies the Hawking-Page phase transition from branch 1 to branch 3 at this point. Furthermore, for $\lambda>\lambda_{c}$ there is only one large black hole configuration with positive heat capacity, 

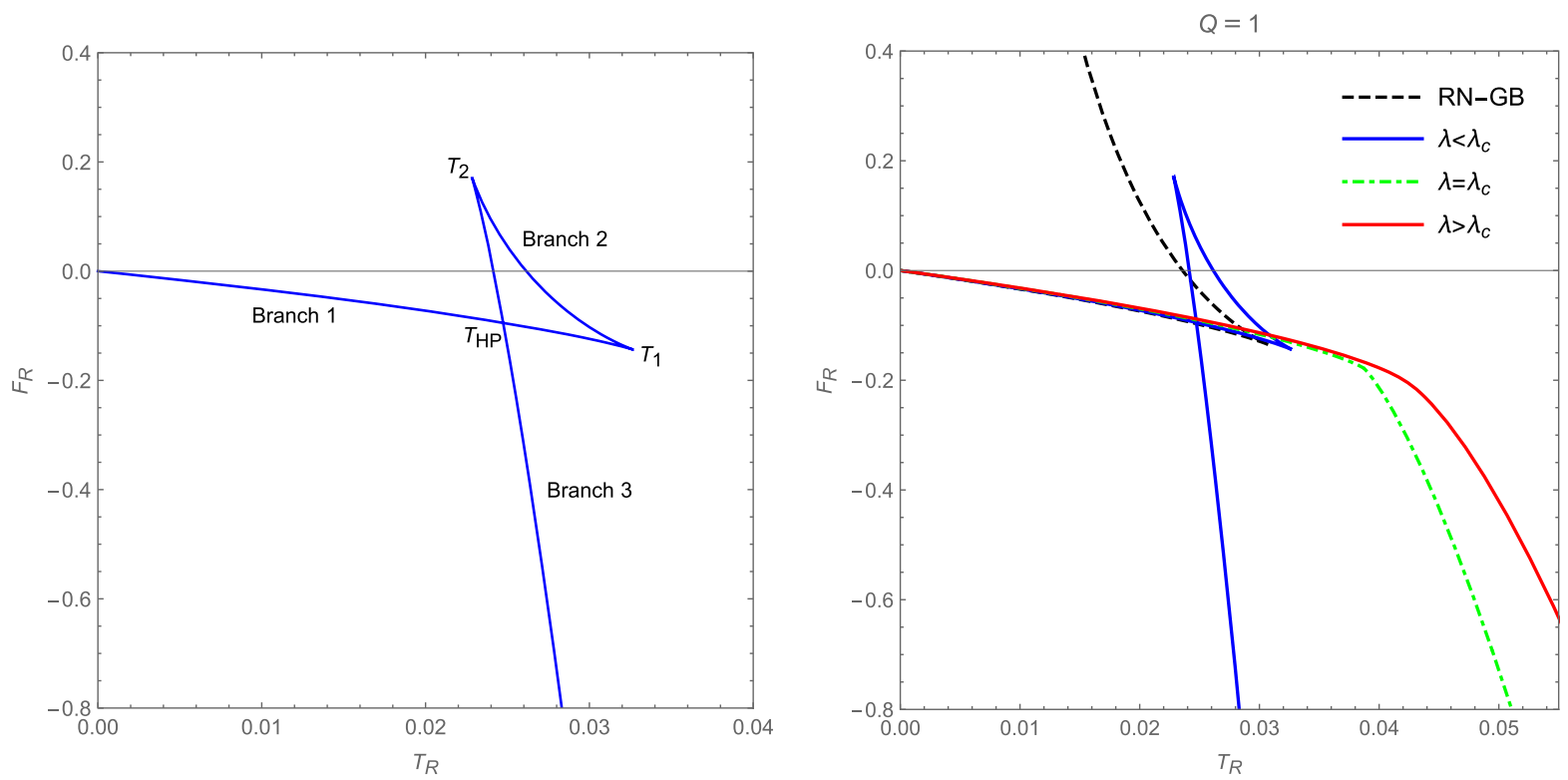

FIG. 5. Left: Branch 1, 2, and 3 of the RN-flat are shown in the swallow-tail-shape graph of the Helmholtz free energy $F_{R}$ versus the Rényi temperature $T_{R}$ at a fixed charge $Q=1$ and $0<\lambda<\lambda_{c}$. Right: Graphs of $F_{R}$ versus $T_{R}$ at different values of $\lambda$ are shown with the graph of that of the RN-flat via GB statistics $(\lambda=0$, dashed). The discussion about these graphs can be seen in the text.

therefore, it is thermodynamically stable. The graphs of the free energy versus Rényi temperature at different values of $\lambda$ are shown in Fig. 5 (right).

\section{THE EMERGENCE OF VAN DER WAALS PHASE TRANSITION}

In the extended phase space approach with the GB statistics, a complete analogy between VdW liquid and RNAdS black holes in canonical ensemble was established by identifying the thermodynamic pressure with cosmological constant $P=-\frac{\Lambda}{8 \pi}$. However, in the asymptotically flat spacetime, the thermodynamic pressure $P=0$ and the black hole phase transition in the $F-T$ plane is not in the VdW type. As discussed in the previous section, the $\lambda$ parameter can give an effect to the thermodynamic description in the same way as the existence of the negative cosmological constant $\Lambda$. In this section, we will derive a consistent Smarr formula for Rényi statistics and show the results that the phase structure of a $\mathrm{RN}$-flat black hole through "Rényi extended phase space" can have the critical behavior like the VdW liquid-gas system in the canonical ensemble.

\section{A. Generalized Smarr formula}

To achieve this, we will extend the usual Smarr formula in terms of the Rényi entropy and its corresponding temperature instead of Bekenstein-Hawking entropy and Hawking temperature. In four-dimensional RN-flat black holes, the Smarr formula is [41]

$$
M=2 T_{H} S_{\mathrm{BH}}+\Phi Q .
$$

Although the parameter $\lambda$ can be a large negative real number, we consider the black hole here with the assumption that there should be a small deviation from the conventional GB entropy due to the effect of nonextensivity corresponding to $0<\lambda \ll 1$. From the Smarr formula above, we can use the relations

$$
T_{H}=\frac{T_{R}}{e^{\lambda S_{R}}} \quad \text { and } \quad S_{\mathrm{BH}}=\frac{e^{\lambda S_{R}}-1}{\lambda},
$$

where they are derived from (28) and (29), to rewrite [41] in the Rényi description. In addition to this, we can expand the first term of [41] in the power series of the $\lambda$ parameter to obtain

$$
\begin{aligned}
M & =2 T_{R} S_{R}-\lambda T_{R} S_{R}^{2}+\Phi Q+O\left(\lambda^{2}\right), \\
& =2 T_{R} S_{R}-\frac{\lambda\left(1-\Phi^{2}\right)}{4} \pi r_{+}^{3}+\Phi Q+O\left(\lambda^{2}\right) .
\end{aligned}
$$

To obtain the second term of the last line, we have substituted $T_{R}$ from (30) and expanded $S_{R}=\pi r_{+}^{2}-$ $\frac{1}{2} \lambda \pi^{2} r_{+}^{4}+O\left(\lambda^{2}\right)$. Then, from the second term of (49), we can identify

$$
P=\frac{3 \lambda\left(1-\Phi^{2}\right)}{32}, \quad V=\frac{4}{3} \pi r_{+}^{3} .
$$

Therefore, the lowest order approximation of $\lambda$ in (49) can be written in the form

$$
M=2 T_{R} S_{R}-2 P V+\Phi Q .
$$


We call this relation here a modified Smarr formula via Rényi statistics. Obviously, we have suggested that the new term in the Smarr formula is the product of thermodynamic pressure $P$ and the thermodynamic volume $V$. The thermodynamic volume is a conjugate quantity to the pressure, which can be obtained through the standard relation $V=\left(\frac{\partial M}{\partial P}\right)_{\Phi, S_{R}}$ at the leading order of $\lambda$. Hence, it is not a geometric spherical volume with horizon radius $r_{+}$.

Typically, the black hole mass corresponds to the internal energy in conventional black hole thermodynamics. However, this thermodynamical interpretation could be changed when we consider the nonextensive thermodynamics from the Rényi statistics. Recall that the first law of black hole thermodynamics from the standard GB statistics can be written in the form

$$
\delta M=T_{H} \delta S_{\mathrm{BH}}+\Omega \delta J+\Phi \delta Q .
$$

It is interesting to ask a question about what the first law of black hole thermodynamics is in the framework of Rényi statistics. To address this, we substitute (48) into (52) in the case of zero-charge and nonrotating, for simplicity, then we arrive at the relation

$$
\begin{aligned}
\delta M & =T_{H} \delta S_{\mathrm{BH}} \\
& =\frac{T_{R}}{e^{\lambda S_{R}}} \delta\left(\frac{e^{\lambda S_{R}}-1}{\lambda}\right), \\
& =T_{R} \delta S_{R}+\frac{1}{8} \pi r_{+}^{3} \delta \lambda+\mathcal{O}\left(\lambda^{2}\right), \\
& \approx T_{R} \delta S_{R}+V \delta P
\end{aligned}
$$

where $P=\frac{3}{32} \lambda$ and $V=\frac{4}{3} \pi r_{+}^{3}$. Our result shows that the mass of black hole $M$ is a function of $S_{R}$ and $P$, therefore, it should be interpreted as the enthalpy $H\left(S_{R}, P\right)$ rather than the internal energy $E\left(S_{R}, V\right)$. This is, in a way, similar to the results from the extended phase space approach [28]. As it is well known, these two quantities can be related through the Legendre transformation $E=H-P V$, and we can then obtain the first law of thermodynamics, in the Rényi thermodynamics, as

$$
\delta E=T_{R} \delta S_{R}-P \delta V,
$$

where the internal energy $E$ is now defined as the black hole mass $M$ subtracted by $P V$.

\section{B. The equation of state}

Typically, in a thermodynamic system, the matter changes its temperature when its microscopic components emit or absorb photons. In the same way, a black hole can change its temperature through gaining or losing its masses. By this analogy, we may assume that the black hole could have its own microstate carrying the degrees of freedom, as well. To describe the black hole microscopic structure, each individual microstate of the black hole has been proposed to contain $\gamma$ Planck area pixels of event horizon surface [35]. Thus, the total number of degrees of freedom is

$$
N=\frac{A}{\gamma l_{p}^{2}},
$$

where $l_{p}=\sqrt{\frac{\hbar G}{c^{3}}}$ is the Planck length, and $l_{p}^{2}$ is the area of one Planck area pixel. We can say that each individual constituent of a black hole has "specific volume." Using (50) and (55), the black hole has the specific volume of the form

$$
v=\frac{V}{N}=\frac{\gamma l_{p}^{2}}{3} r_{+} .
$$

Note that the specific volume $v$ scales linearly in the horizon radius $r_{+}$of the black holes. In the remaining of this section, we will explore about the equation of state and thermal phase diagram from this approach in both grand canonical and canonical ensembles.

\section{Grand canonical ensemble}

With the macroscopic perspective, the equation of state of the RN-flat black hole in the fixed $\Phi$ ensemble can be obtained from the formula of $P, v, T_{R}$. Summarizing from (30), (50), and (56), we now have

$$
\begin{aligned}
T_{R} & =\frac{\left(1-\Phi^{2}\right)\left(1+\lambda \pi r_{+}^{2}\right)}{4 \pi r_{+}}, \\
P & =\frac{3 \lambda\left(1-\Phi^{2}\right)}{32}, \\
v & =\frac{8}{3} r_{+} .
\end{aligned}
$$

Combining these equation with the elimination of $\lambda$, we will arrive at the equation of state

$$
P=\frac{T_{R}}{v}-\frac{2\left(1-\Phi^{2}\right)}{3 \pi v^{2}},
$$

where we have used $l_{p}=1$ and $\gamma=8$ in (58). As will be clear later, choosing the value of $\gamma$ like this allows us to obtain the universal constant $Z_{c}$ consistent with that in $\mathrm{VdW}$ fluid. Remark that the equation of state is written in the form of the specific volume $v$, which is proportional to $r_{+}$rather than the thermodynamic volume $V$. In the grand canonical ensemble, the relations between $P$ and $v$ in isothermal process with different values of $T_{R}$ are plotted at $\Phi=0.5,0.7$ in Fig. 6. By solving the condition $\left(\frac{\partial P}{\partial v}\right)_{T_{R}, \Phi}=0$, the pressure has a maximum value $P_{\max }$ at $v_{c}$ as 

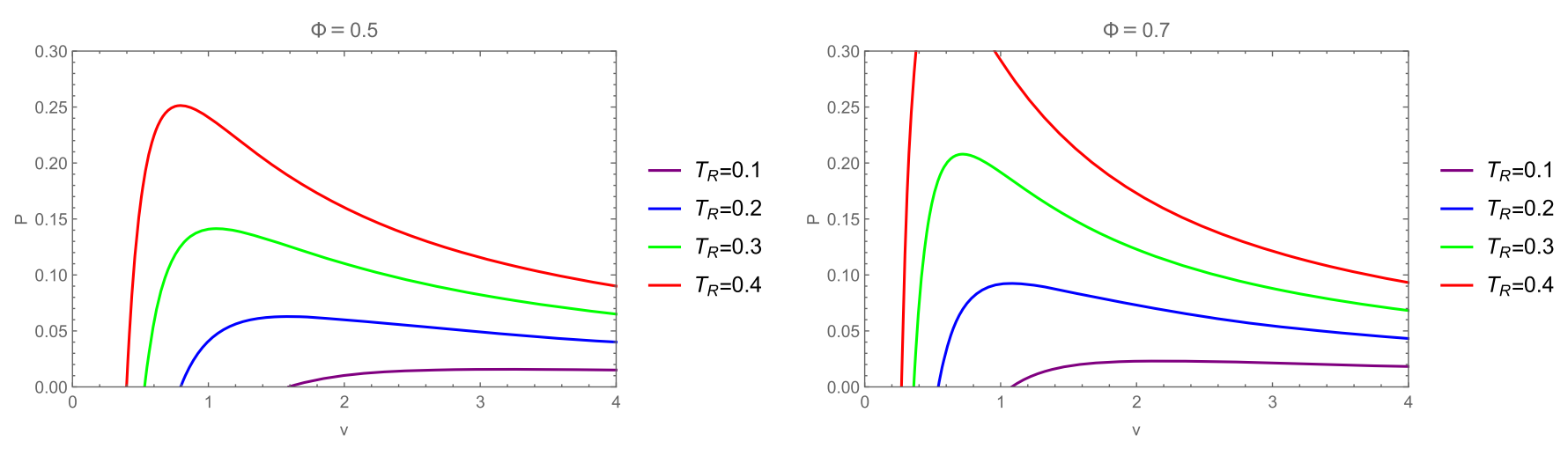

FIG. 6. $\quad P-v$ diagram of charged black holes for fixed potential $\Phi=0.5$ (left) and $\Phi=0.7$ (right). In both graphs, the temperature of isotherms decreases from top to bottom. For a given temperature, there are two branches of black holes, one is the small black hole $(P$ increases as $v$ increases) and the other is the large black hole ( $P$ decreases as $v$ increases).

$$
P_{\max }=\frac{3 \pi T_{R}^{2}}{8\left(1-\Phi^{2}\right)}, \quad v_{c}=\frac{4\left(1-\Phi^{2}\right)}{3 \pi T_{R}}
$$

For a given temperature, there are two black hole configurations below a maximum pressure $P_{\max }$ corresponding with small and large black holes when $v<v_{c}$ and $v>v_{c}$, respectively. The small black hole phase is unstable because $P$ increases as $v$ increases, therefore, the compression coefficient is negative. In the large black hole phase, the compression coefficient is positive because $P$ decreases as $v$ increases, hence it is thermodynamically stable. We can find an "ideal gas" behavior for large black holes at high temperature. However, as seen from (59), the second derivative $\left(\frac{\partial^{2} P}{\partial v^{2}}\right)_{T_{R}, \Phi}$ cannot vanish at $v_{c}$. This means that there is no critical behavior for the RN-flat black hole in the grand canonical ensemble.

\section{Canonical ensemble}

For the canonical ensemble, it has been known that the transition of small/large RN-AdS black holes in extended phase space is analogous to the liquid/gas phase transition of the VdW type. Therefore, it is interesting to see whether the $P-v$ criticality appears or not for the Rényi extended phase space approach in this ensemble. As in the previous section, we identify the macroscopic quantities as

$$
\begin{aligned}
T_{R} & =\frac{\left(r_{+}^{2}-Q^{2}\right)\left(1+\lambda \pi r_{+}^{2}\right)}{4 \pi r_{+}^{3}}, \\
P & =\frac{3 \lambda}{32}\left(1-\frac{Q^{2}}{r_{+}^{2}}\right), \\
v & =\frac{8}{3} r_{+},
\end{aligned}
$$

where we have used $\Phi=\frac{Q}{r_{+}}, l_{p}=1$ and $\gamma=8$. The equations of state in terms of $P, v$, and $T_{R}$ can be written in the form

$$
P=\frac{T_{R}}{v}-\frac{2}{3 \pi v^{2}}+\frac{128 Q^{2}}{27 \pi v^{4}} .
$$

At a fixed value of $Q$, this formula indicates that the $P-v$ diagram shows a critical behavior like the $\mathrm{VdW}$ liquid-gas system, as shown in Fig. 7. The phase structure can be critically changed when the temperature crosses the critical point, which is the point of inflection with these two conditions

$$
\left(\frac{\partial P}{\partial v}\right)_{T_{R}, Q}=0, \quad\left(\frac{\partial^{2} P}{\partial v^{2}}\right)_{T_{R}, Q}=0 .
$$

Solving these conditions leads to the critical thermodynamic quantities of the charged black holes as follows:

$$
\begin{aligned}
P_{c} & =\frac{1}{128 \pi Q^{2}}, \\
v_{c} & =8 \sqrt{\frac{2}{3}} Q, \\
T_{c} & =\frac{1}{3 \sqrt{6} \pi Q} .
\end{aligned}
$$

These formulas of critical values lead us to the critical compressibility factor

$$
Z_{c} \equiv \frac{P_{c} v_{c}}{T_{c}}=\frac{3}{8},
$$

which is a universal constant in the sense that it is independent of a black hole's mass and charge. Interestingly, this universal constant from our results has the same value as in the VdW fluid. Remark that $Z_{c}=\frac{3}{8}$ exactly when the factor $\gamma=8$. This in turn indicates that for consistent values of $Z_{c}$, we need 1 degree of freedom of the black hole to consist of 8 Planck area pixels on the event horizon in the Rényi extended phase space model. In other 

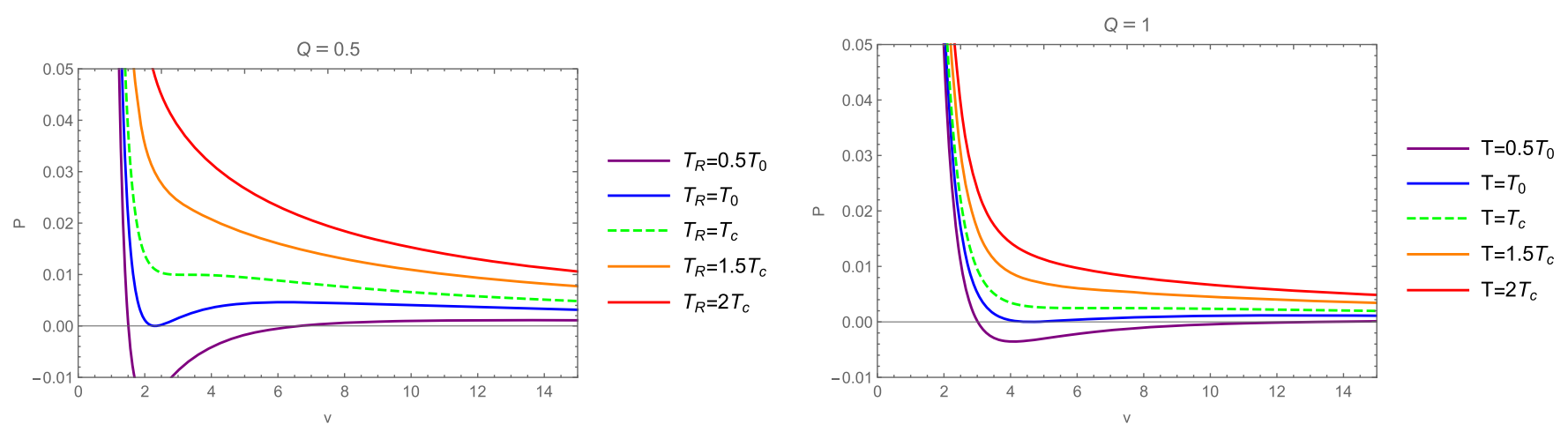

FIG. 7. $P-v$ diagram of charged black holes for fixed charge $Q=0.5$ (left) and $Q=1$ (right). In both graphs, the temperature of isotherms decreases from top to bottom. The dashed green line is denoted as the critical isotherm $T=T_{c}$. The lower solid lines correspond to small temperatures $T<T_{c}$, which have three branches of black holes, one unstable $(P$ increases as $v$ increases) and two stable ( $P$ decreases as $v$ increases) configurations. The upper solid lines are in high temperature limit $T>T_{c}$, analogous to the ideal gas phase of VdW system.

words, the value of the critical compressibility factor depends on the number of Planck area pixels, which represents 1 degree of freedom of the black hole. For the RN-AdS black hole extended phase space, it is, however, necessary to choose $\gamma=6$ to obtain the result $Z_{c}=\frac{3}{8}$ [29].

The $P-v$ diagram in Fig. 7 is the same as in the $\mathrm{VdW}$ liquid-gas system. The left and right graphs of this figure correspond to the case of fixed charge $Q=0.5,1$, respectively. The critical isothermal curve is depicted by the green dashed line. For $T_{R}<T_{c}$, there is a small black hole phase at small value $v$ corresponding to the liquid phase while there is a large black hole phase at large value $v$ corresponding to the gas phase. These two stable configurations have a positive compression coefficient ( $P$ decreases as $v$ increases). We also have the unstable region with a negative compression coefficient ( $P$ increases as $v$ increases) between these two regions at which the black hole of intermediate size can represent the mixture of liquid and gas phases. Moreover, there is a minimum temperature $T_{0}$ given by

$$
T_{0}=\frac{1}{6 \sqrt{3} \pi Q}
$$

associated with $P=0$ at $v_{0}=\frac{8 Q}{\sqrt{3}}$, as seen in the blue solid line in Fig. 7. For $T<T_{0}$, the black hole pressure becomes negative at some specific volume $v$. In a high temperature case $T_{R}>T_{c}$, the black holes behave like an ideal gas and no phase transition occurs.

\section{CONCLUSION}

In this paper, we investigate the thermodynamics of charged black holes in asymptotically flat spacetime or RNflat via an alternative Rényi entropy both in the grand canonical and canonical ensembles. Applying the Rényi statistics, our results indicate that it is possible, with $0<\lambda<1$, to have the small and large black hole branches in the grand canonical ensemble while this cannot occur in the GB statistics approach. Moreover, we have shown that the Hawking-Page phase transition between thermal radiation and large black holes in RN-flat can happen and crucially depend on the $\lambda$ parameter.

For the canonical ensemble, in contrast with the grand canonical case, there is a critical behavior such that a small/ large black hole first-order phase transition can occur when $\lambda<\lambda_{c}$. Above the critical value of the Renyi parameter $\lambda_{c}$, this phase transition disappears and the large black hole phase is only the remaining phase that is possible. This thermal behavior is, in the same way, as that of the charged black hole in AdS space with the standard GB statistics. While the conjectured equivalence between the thermodynamics of asymptotically flat black holes via Rényi statistics and that of AdS black holes via GB statistics have been investigated through the works of considering Schwarzschild and Kerr black holes by Czinner et al. $[23,24]$, our results on considering charged black holes also support this proposed equivalence. Note that we discuss, in detail, the thermodynamic stability of black holes through comparing the size of black holes with the nonextensivity length $L_{\lambda} \equiv 1 / \sqrt{\pi \lambda}$ in the Appendix.

Importantly, we explore further in this paper to find more evidence to support the above mentioned equivalence through the consideration of our setup in a modified extended phase space approach. A consistent Smarr formula for Rényi statistics is derived. We suggest that the $\lambda$ parameter contributes to the thermodynamic pressure $P$ for black holes, as shown in (50). Then, the quantity conjugate to the pressure may be quantified as a thermodynamic volume of black holes. This result shows that the black hole mass represents the enthalpy rather than the internal energy. Introducing a specific volume, which is thermodynamic volume of black holes for each degree of freedom, we write 
the equation of state and study the thermal behavior of charged black holes in flat space with nonzero $\lambda$ parameter. In the $P-v$ diagram, we find that its thermal phase structure in the canonical ensemble has the VdW-like phase transition. Intriguingly, taking $\gamma=8$, we obtain the critical compressibility factor $Z_{c}=\frac{3}{8}$ as in the $\mathrm{VdW}$ equation of state. The consideration of our setup in the extended phase space approach gives the result corresponding to the VdW-like phase structure found in the case of AdS charged black holes via the GB statistics. As a consequence, this gives more supporting evidence of the existence of the equivalence, but it is in a different way from the aforementioned works of Czinner et al.

The microstates of a self-gravitating system could be correlated, in some ways, among themselves within the system and also with those in the environment. We suggest that, in nonextensive description, some energy density emerged from the nontrivial correlation between the black hole and heat bath may be encoded in the nonextensive parameter $\lambda$. We discuss this in more detail in the Appendix. This emergent energy density from nonextensive nature induces a pressure to the black holes in the same way as $|\Lambda|$ plays a role in the case of AdS black holes through the extended phase space approach. In other words, the nonextensive parameter $\lambda$ then behaves effectively as the cosmological constant $|\Lambda|$, which emerges from the nontrivial thermodynamical behavior.

If the nonextensivity allows us to have the emergent energy from the correlations, as suggested above, the vacuum around the black hole can gain some energy density from the system-environment correlations resulting from long-range gravitation. Considering in the Rényi extended phase space, using (53) and (54), we can associate the black hole mass to the enthalpy, i.e., $M=E+P V$, rather than the internal energy $E$. This has a physical implication that the creation of a black hole mass costs not only the internal energy, but also the additional energy responsible to the correlations.

As it seems that the thermodynamic behaviors of these two physical systems with different statistics are possible to be equivalent, our results also indicate that there should be some connections between the Rényi parameter $\lambda$ and the absolute value of AdS space cosmological constant $|\Lambda|$. As shown in (8) and (50), both $\lambda$ and $|\Lambda|$ play the roles as thermodynamic pressure up to some constants. This connection could guide us to understand more about the physical meaning of the Rényi parameter $\lambda$, and the running of this parameter might work, in some ways, similar to the running of $|\Lambda|$ in AdS black holes with the GB statistics. However, this is beyond the scope of this paper, thus it may be studied in future works.

\section{ACKNOWLEDGMENTS}

We are grateful to Pitayuth Wongjun, Supakchai Ponglertsakul, Sirachak Panpanich, Krai Cheamsawat, and Tanapat Deesuwan for helpful discussions. This work has been supported by the Petchra Pra Jom Klao Ph.D. Research Scholarship from King Mongkut's University of Technology Thonburi. Moreover, W. Liewrian would like to thank the Ministry of Higher Education, Science, Research and Innovation, Thailand, for financial supporting this research by grant fund under the Thailand Center of Excellence in Physics.

\section{APPENDIX: NONEXTENSIVE EFFECT ON BLACK HOLE STABILITY}

Taking into account a nonextensive effect, one can show the emergence of an additional energy density from the presence of nonzero Rényi parameter $\lambda$. In a microcanonical ensemble of Schwarzschild black holes together with thermal radiation in asymptotically flat, we have a fixed total energy density $E=E_{\mathrm{bh}}+E_{r}=$ const, where $E_{\mathrm{bh}}$ and $E_{r}$ stand for the energy density of black holes and thermal radiation, respectively. Basically, the thermal equilibrium exists when the system is in the most probable state. This results in the maximum value of the total entropy $S=S_{\mathrm{bh}}+S_{r}$. Hence, the conditions of thermal equilibrium consist of

$$
\frac{\partial S}{\partial E_{r}}=0, \quad \text { and } \quad \frac{\partial^{2} S}{\partial E_{r}^{2}}<0
$$

The first condition gives

$$
\begin{aligned}
0 & =d S=d S_{\mathrm{bh}}+d S_{r} \\
& =\frac{d E_{\mathrm{bh}}}{T_{\mathrm{bh}}}+\frac{d E_{r}}{T_{r}} \\
& =\left(\frac{1}{T_{\mathrm{bh}}}-\frac{1}{T_{r}}\right) d E_{\mathrm{bh}},
\end{aligned}
$$

where we have used the conservation law of energy $d E_{r}=-d E_{\mathrm{bh}}$. The above equation gives us the condition $T_{\mathrm{bh}}=T_{r}$, as it should be. On the other hand, the second condition reads

$$
\begin{aligned}
\frac{\partial^{2} S}{\partial E_{r}^{2}} & =\frac{\partial^{2} S_{\mathrm{bh}}}{\partial E_{\mathrm{bh}}^{2}}+\frac{\partial^{2} S_{r}}{\partial E_{r}^{2}} \\
& =-\frac{1}{T_{\mathrm{bh}}^{2}} \frac{\partial T_{\mathrm{bh}}}{\partial E_{\mathrm{bh}}}-\frac{1}{T_{r}^{2}} \frac{\partial T_{r}}{\partial E_{r}} \\
& =-\frac{1}{T^{2}}\left(\frac{1}{C_{\mathrm{bh}}}+\frac{1}{C_{r}}\right)<0 .
\end{aligned}
$$

Obviously, we obtain the inequality condition on the heat capacity in the form

$$
\frac{1}{C_{\mathrm{bh}}}+\frac{1}{C_{r}}>0
$$


Using $E_{r}=\sigma \tilde{V} T_{r}^{4}$, we have the heat capacity of a radiation filled in volume $\tilde{V}$ as

$$
C_{r}=4 \sigma \tilde{V} T_{r}^{3}
$$

Using (39), (45), (A5), and setting the electric charge $Q=0$ for simplicity, the second condition (A4) becomes

$$
-\frac{1-\lambda \pi r_{+}^{2}}{2 \pi r_{+}^{2}}+\frac{1}{4 \sigma \tilde{V} T^{3}}>0 .
$$

This condition of thermal equilibrium relates the volume $\tilde{V}$ of the space filled with thermal radiation, the black hole's size $r_{+}$, and nonextensive parameter $\lambda$. Interestingly for the black hole in the heat bath of infinitely large volume $\tilde{V} \rightarrow \infty$, we have

$$
\lambda>\frac{1}{4 \pi M_{\mathrm{bh}}^{2}},
$$

where we have used $M_{\mathrm{bh}}=\frac{r_{+}}{2}$. The black holes can be in thermal equilibrium with an infinite heat reservoir when the black hole mass satisfies the above inequality. Notice that in the geometric unit $\lambda$ has the dimension of (length $)^{-2}$. As defined in (50), the pressure in the form $P \sim \lambda$ thus has the dimension of energy density. In the Rényi version of extended phase space, the appearance of an extra term $V \delta P$ in (53) can be thought of as the contribution from the nonextensivity in the form of an additional energy. With this point of view, we can also think that this additional energy contributes as the term with $\lambda$ in (A6).

Interestingly, (A7) can be rewritten in the form

$$
r_{+}>L_{\lambda}
$$

where $L_{\lambda} \equiv 1 / \sqrt{\pi \lambda}$. This indicates that the black hole in asymptotically flat spacetime can be stable only if the event horizon radius is larger than the characteristic length scale $L_{\lambda}$. The presence of this nonextensivity length implies that there is a particular value of gravitational energy beyond which the conventional statistical mechanics is no longer applicable. This is because the nonextensive effect turns out to take an important role in describing black hole thermodynamics when $r_{+}$is larger than $L_{\lambda}$.
[1] J. D. Bekenstein, Black holes and entropy, Phys. Rev. D 7, 2333 (1973).

[2] S. W. Hawking, Particle creation by black holes, Commun. Math. Phys. 43, 199 (1975).

[3] J. M. Bardeen, B. Carter, and S. W. Hawking, The four laws of black hole mechanics, Commun. Math. Phys. 31, 161 (1973).

[4] S. W. Hawking and D. Page, Thermodynamics of black holes in anti-de Sitter space, Commun. Math. Phys. 87, 577 (1983).

[5] D. Lynden-Bell, Negative specific heat in astronomy, physics and chemistry, Physica (Amsterdam) 263A, 293 (1999).

[6] W. Thirring, System with negative specific heat, Z. Phys. 235, 339 (1970).

[7] J. W. Gibbs, Elementary Principles in Statistical Mechanics Developed with Special Reference to the Rational Foundation of Thermodynamics/by J. Willard Gibbs (C. Scribner, New York, 1902).

[8] P. T. Landsberg, Thermodynamics and Statistical Mechanics (Oxford University Press, New York, 1978).

[9] P. Landsberg, Is equilibrium always an entropy maximum?, J. Stat. Phys. 35, 159 (1984).

[10] C. Tsallis, Possible generalization of Boltzmann-Gibbs statistics, J. Stat. Phys. 52, 479 (1988).

[11] C. Tsallis, Introduction to Nonextensive Statistical Mechanics: Approaching a Complex World (Springer Science and Business Media, New York, NY, USA, 2009).
[12] G. Hooft, Dimensional reduction in quantum gravity, Conf. Proc. C 930308, 284 (1993).

[13] L. Susskind, The world as a hologram, J. Math. Phys. (N.Y.) 36, 6377 (1995).

[14] J. Maldacena, The large N limit of superconformal field theories and supergravity, Int. J. Theor. Phys. 38, 1113 (1999).

[15] E. Witten, Anti-de Sitter space and holography, Adv. Theor. Math. Phys. 2, 253 (1998).

[16] E. Witten, Anti-de Sitter space, thermal phase transition, and confinement in gauge theories, Adv. Theor. Math. Phys. 2, 505 (1998).

[17] M. Srednicki, Entropy and Area, Phys. Rev. Lett. 71, 666 (1993).

[18] S. Abe, General pseudoadditivity of composable entropy prescribed by the existence of equilibrium, Phys. Rev. E 63, 061105 (2001).

[19] M. Nauenberg, The critique of $q$ entropy for thermal statistics, Phys. Rev. E 67, 036114 (2003).

[20] T.S. Biró and P. Ván, Zeroth law compatibility of nonadditive thermodynamics, Phys. Rev. E 83, 061147 (2011).

[21] A. Rényi, On the dimension and entropy of probability distribution, Acta Math. Acad. Sci. Hung. 10, 193 (1959).

[22] C. Tsallis and L. J. L. Cirto, Black hole thermodynamical entropy, Eur. Phys. J. C 73, 2487 (2013).

[23] V. G. Czinner and H. Iguchi, Rényi entropy and the thermodynamic stability of black holes, Phys. Lett. B 752, 306 (2016). 
[24] V. G. Czinner and H. Iguchi, Thermodynamics, stability and Hawking-Page transition of Kerr black holes from Rényi statistics, Eur. Phys. J. C 77, 892 (2017).

[25] A. Chamblin, R. Emparan, C. V. Johnson, and R. C. Myers, Charged AdS black holes and catastrophic holography, Phys. Rev. D 60, 064018 (1999).

[26] A. Chamblin, R. Emparan, C. V. Johnson, and R. C. Myers, Holography, thermodynamics and fluctuations of charged AdS black holes, Phys. Rev. D 60, 104026 (1999).

[27] P. Burikham and C. Promsiri, The mixed phase of charged AdS black holes, Adv. High Energy Phys. 2016, 5864672 (2016).

[28] D. Kastor, S. Ray, and J. Traschen, Enthalpy and the mechanics of AdS black holes, Classical Quantum Gravity 26, 195011 (2009).

[29] D. Kubiznak and R. Mann, P-v criticality of charged AdS black holes, J. High Energy Phys. 07 (2012) 033.

[30] D. Kubiznak and R. Mann, Black hole chemistry: Thermodynamics with Lambda, Classical Quantum Gravity 34, 063001 (2017).

[31] S. Gunasekaran, R. Mann, and D. Kubiznak, Extended phase space thermodynamics for charged and rotating black holes and Born-Infeld vacuum polarization, J. High Energy Phys. 11 (2012) 110.

[32] R. G. Cai, L. M. Cao, L. Li, and R. Q. Yang, P-V criticality in the extended phase space of Gauss-Bonnet black holes in AdS space, J. High Energy Phys. 09 (2013) 005.
[33] S. Hendi, S. Panahiyan, and B. Eslam, Panah Extended phase space of black holes in Lovelock gravity with nonlinear electrodynamicss, Prog. Theor. Exp. Phys. 2015, 103E01 (2015).

[34] C. Niu, Y. Tian, and X. N. Wu, Critical phenomena and thermodynamic geometry of RN-AdS black holes, Phys. Rev. D 85, 024017 (2012).

[35] S. W. Wei and Y.X. Liu, Insight into the Microscopic Structure of an AdS Black Hole from a Thermodynamical Phase Transition, Phys. Rev. Lett. 115, 111302 (2015).

[36] S. W. Wei, Y. X. Liu, and R. B. Mann, Ruppeiner geometry, phase transitions, and the microstructure of charged AdS black holes, Phys. Rev. D 100, 124033 (2019).

[37] M. Cadoni, E. Franzin, and M. Tuveri, Van der Walls-like behaviour of charged black holes and hysteresis in the dual QFTs, Phys. Lett. B 768, 393 (2017).

[38] F. Bouchet and J. Barré, Classification of phase transitions and ensemble inequivalence, in systems with long range interactions, J. Stat. Phys. 118, 1073 (2005).

[39] D. J. Gross, M. J. Perry, and L. G. Yaffe, Instability of flat space at finite temperature, Phys. Rev. D 25, 330 (1982).

[40] R. Penrose, Gravitational Collapse and Spacetime Singularity, Phys. Rev. Lett 14, 57 (1965).

[41] L. Smarr, Mass Formula for Kerr Black Holes, Phys. Rev. Lett. 30, 71 (1973). 